\title{
Active Antennas for Space Applications
}

\author{
Stefano Selleri ${ }^{1}$ and Giovanni Toso ${ }^{2}$ \\ ${ }^{1}$ Department of Electronics and Telecommunications, University of Florence, Via C. Lombroso 6/17, 50134 Firenze, Italy \\ ${ }^{2}$ Antenna and Sub-Millimeter Wave Section, Electromagnetic Division, European Space Agency, ESA-ESTEC, Keplerlaan 1, \\ PB 299, 2200 AG Noordwijk, The Netherlands
}

Correspondence should be addressed to Stefano Selleri, stefano.selleri@unifi.it

Received 14 April 2009; Accepted 14 April 2009

Copyright () 2009 S. Selleri and G. Toso. This is an open access article distributed under the Creative Commons Attribution License, which permits unrestricted use, distribution, and reproduction in any medium, provided the original work is properly cited.

Phased arrays produce beams that can be modified, scanned or shaped electronically. An active array antenna system is defined as an array in which all or part of the elements are equipped with their own transmitter or receiver, or both [1].

Active antennas (AAs) are devices combining a radiating subsystem as well as backing active circuits in tight integration, drastically reducing the complexity of the matching networks and filters by carefully designing the radiating element so as to provide the proper load for the active elements.

Although the idea of an AA is not new [2-7], its extended impact on telecommunication industry is recent and there is a large interest, both from researchers and industries, in novel fields of application. These spans from satellite [8] to biomedical applications [9], to radioastronomy $[10,11]$ and, of course, to personal communication systems, wireless local networks, and RF identification. Recent research threads, selected among the referred journal publications of the last two years, investigate possible band widening $[12,13]$, polarization diversity [14], applications in MIMO systems [15], and novel radiators [16, 17].

The current lead applications of AA are large electronically scanned arrays $[18,19]$, and this special issue is indeed focused on this broad topic, with particular attention to satellite applications.

Indeed arrays of active antennas, or active arrays, are well suited for mobile terminals requiring dynamic satellite tracking. The most common approach toward achieving fastbeam scanning is through the integration of monolithic microwave integrated circuit (MMIC) phase shifters, lownoise amplifiers, and solid state power amplifiers with the antenna elements. In some cases, hybrid electro/mechanical arrays combining mechanical steering with electrical steering/shaping are considered. This architecture is often used to reduce the number of active control elements by limiting the electrical scanning in only one plane. This is often the case for mobile user terminals where azimuth scanning is performed by mechanical rotation and elevation agility is realized by a linear phased array.

Active phased arrays are well known for being complex and expensive antennas and, as a consequence, only few space missions exploited this type of antenna. This is not the case for ground and airborne radars, where major funding, mainly from the US defense programs, has led to large-scale production of T/R modules reaching a goal of a $\$ 100$ cost per module already in the 80s. Unfortunately, this background is hardly reusable for space missions, due to the completely different requirements and operational constraints. One interesting exception is the IRIDIUM satellite system, where the main mission antenna has been conceived essentially reusing $\mathrm{T} / \mathrm{R}$ technology previously developed for radar applications [20].

Another factor limiting a wider application of antenna phased arrays in space missions is linked to their large mass and high power dissipation, especially for the large active phased array configurations. This generates the need for new ultralight technologies and thermal hardware. With the progress in developments of more efficient T/R modules, thermal aspects may become less critical, but the improvement on this parameter is not as quick as expected.

During the past 20 years, the basic radiating elements and feeding lines present in phased array systems have evolved with a variety of microstrip, stripline, and other printed 


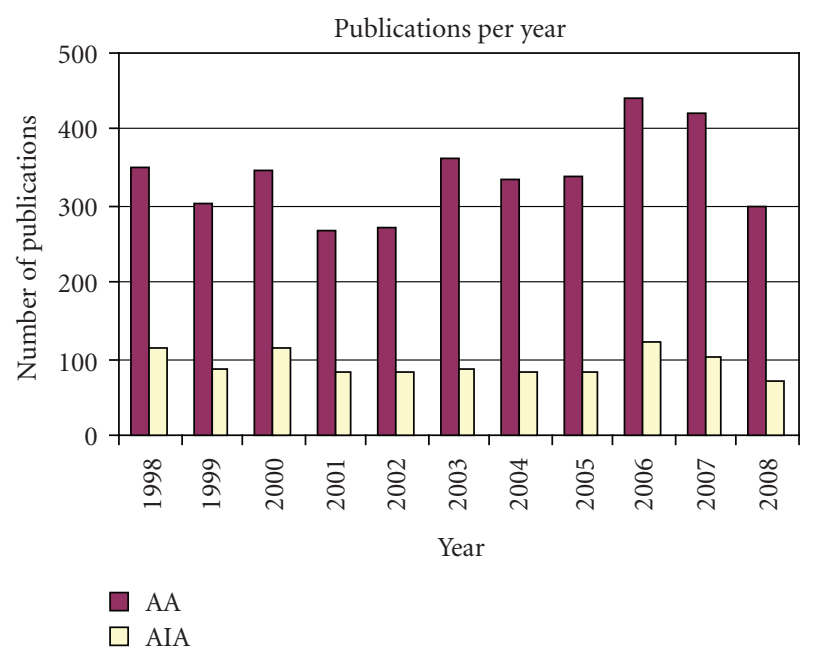

FIgURE 1: Number of publications per year, (journal, conferences and books), on active antennas (purple) and on active integrated antennas (yellow).

radiators replacing the traditional dipoles, slots, or horns, fed by coaxial lines or waveguides. Developments in monolithic and other fabrication processes allowed for the automated production of phased arrays with a high integration level and not by piecewise assembly as in the beginning. Using digital processing or analog devices, the active phased arrays will have time-delay capability to make wideband performance possible. Other important features of array antennas are the distribution of solid-state power amplification with graceful degradation and a lower risk of power handling. Active phased arrays can also be designed to conform to the surface of a spacecraft.

Today phased arrays for space missions are mostly considered when in flight beam reconfigurability or inertialess beam steering is required. However, it is important to note that array antennas may find wider space applications when nongeostationary telecommunication constellations are considered, or as a way to generate very large apertures thanks to digital processing techniques for beamforming.

Active phased array antennas directly radiating or receiving the signal are called "Direct Radiating Arrays" (DRAs). If, on the other hand, they are used to illuminate reflectors the antenna systems are called "Array Fed Reflectors" (AFRs). Feed arrays can compensate for the inherent distortions of large reflectors deployed in space, thanks to techniques monitoring the reflector surface or synthesize a desired radiation pattern by adjusting the feed coefficients and they can assist to move the complete multiple beam pattern over the coverage, therewith handling seasonal deviations in desired scanning/coverages, without moving the satellite thus saving propellant and extending its lifetime.

At last, to give an idea of the relevance of $\mathrm{AA}$ in electromagnetic engineering, a survey has been made over the INSPEC bibliographical database concerning scientific publications addressing active antenna issues and covering the last ten years. Figure 1 shows this survey, discriminating between active antennas in general (purple) and the subclass of active integrated antennas. A steady average of nearly 350

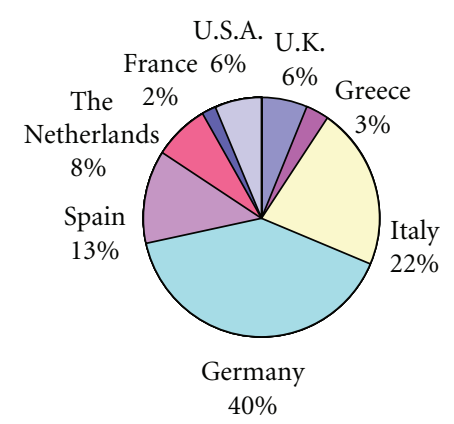

(a)

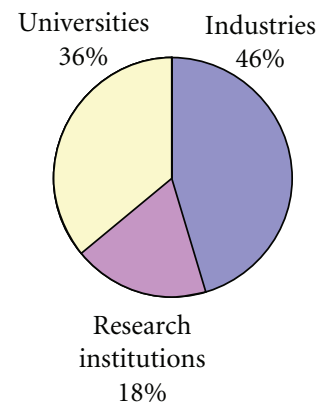

(b)
FIGURE 2: Subdivision of authors by country (a) and subdivision of institutions (b).

papers per year has been published on active antennas in the last decade, showing the large interest of researchers and companies on this topic.

This special issue collects fourteen articles, coming from 63 authors of 22 institutions of 8 countries, worldwide. Figure 2 shows some statistics. The fourteen articles have been ideally subdivided into two parts; the first part gathering contributions which are more oriented to research and leading technology issues, the second part containing more technological and applicative papers. The first author and a very brief description of the content are listed here below.

Part I:

(1) Gaebler et al. present a novel liquid-crystals-based approach to tunable devices for millimeter wave reflectarrays.

(2) Cabria et al. deal with a novel beamsteering technique for reflectarrays.

(3) Theodoros N. Kaifas and John N. Sahalos propose the design of oscillators by exploiting interelement coupling in the array.

(4) Lucci et al. present dielectric resonator antennas harmonic tuning for optimum amplifier loading.

(5) Mailloux et al. exploit polyomino subarrays to achieve superior sidelobe and band performances.

(6) Viganò et al. present a sunflower-like array achieving a quasi-rotationally symmetric pattern with adjustable sidelobes and grating lobes level.

Part II:

(7) Capece describes the COSMO Sky Med SAR array antenna discussing implementation and design issues.

(8) Younis et al. present a comparison between different implementations of digital beamforming SAR.

(9) Bachmann et al. investigate pattern modeling techniques in the TerraSAR-X mission.

(10) Guy investigates the advantages of fully dynamic beam scanning for INMARSAT platform. 
(11) Barba et. al. investigate possible topologies for reducing the beamforming network complexity.

(12) Catalani et al. present a complete design for an airplane-to-satellite active array.

(13) Vincenti Gatti et al. describe two novel design for flat fully-steerable antenna arrays for mobile-to-satellite communications.

(14) Shelley et al. show a low profile antenna for the air or ground terminal mobile-to-satellite link. This latter is a fully mechanically steered antenna but is here included because it provides a reference for the previous two active antenna systems.

Stefano Selleri Giovanni Toso

\section{References}

[1] IEEE Standard Definitions of Terms for Antennas, IEEE Std 145-1993 (Revision of IEEE Std 145-1983).

[2] H. H. Meinke, "Active antennas," Nachrichtentechnische Zeitschrift, vol. 19, pp. 697-705, 1966.

[3] H. A. Wheeler, "Small antennas," IEEE Transactions on Antennas and Propagation, vol. 23, no. 4, pp. 462-469, 1975.

[4] J. Lin and T. Itoh, "Active integrated antennas," IEEE Transactions on Microwave Theory and Techniques, vol. 42, no. 12, pp. 2186-2194, 1994.

[5] J. A. Navarro and K. Chang, Integrated Active Antennas and Spatial Power Combining, John Wiley \& Sons, New York, NY, USA, 1996.

[6] R. A. York and Z. B. Popovic, Active and Quasi-Optical Arrays for Solid-State Power Combining, John Wiley \& Sons, New York, NY, USA, 1997.

[7] A. Mortazwi, T. Itoh, and J. Harvey, Active Antennas and Quasi-Optical Arrays, John Wiley \& Sons, New York, NY, USA, 1998.

[8] C. Mangenot, "Space antenna challenges and roadmaps," in Proceedings of the 29th ESA Antenna Workshop on Multiple Beams and Reconfigurable Antennas, Noordwijk, The Netherlands, April 2007.

[9] S. Jacobsen and $\varnothing$. Klemetsen, "Improved detectability in medical microwave radio-thermometers as obtained by active antennas," IEEE Transactions on Biomedical Engineering, vol. 55, no. 12, pp. 2778-2785, 2008.

[10] S. Jacobsen and $\varnothing$. Klemetsen, "Active antennas in medical microwave radiometry," Electronics Letters, vol. 43, no. 11, pp. 606-608, 2007.

[11] S. W. Ellingson, J. H. Simonetti, and C. D. Patterson, "Design and evaluation of an active antenna for a $29-47 \mathrm{MHz}$ radio telescope array," IEEE Transactions on Antennas and Propagation, vol. 55, no. 3, pp. 826-831, 2007.

[12] D. Segovia-Vargas, D. Castro-Galan, L. E. Garcia-Munoz, and V. Gonzalez-Posadas, "Broadband active receiving patch with resistive equalization," IEEE Transactions on Microwave Theory and Techniques, vol. 56, no. 1, pp. 56-64, 2008.

[13] V. Rizzoli, A. Costanzo, and P. Spadoni, "Computer-aided design of ultra-wideband active antennas by means of a new figure of merit," IEEE Microwave and Wireless Components Letters, vol. 18, no. 4, pp. 290-292, 2008.

[14] G. Yun, "Compact active integrated microstrip antennas with circular polarisation diversity," IET Microwaves, Antennas and Propagation, vol. 2, no. 1, pp. 82-87, 2008.
[15] M. Sadek, A. Tarighat, and A. H. Sayed, "Active antenna selection in multiuser MIMO communications," IEEE Transactions on Signal Processing, vol. 55, no. 4, pp. 1498-1510, 2007.

[16] A. Guraliuc, G. Manara, P. Nepa, G. Pelosi, and S. Selleri, "Harmonic tuning for ku-band dielectric resonator antennas," IEEE Antennas and Wireless Propagation Letters, vol. 6, pp. 568-571, 2007.

[17] L. K. Hady, D. Kajfez, and A. A. Kishk, "Dielectric resonator antenna in a polarization filtering cavity for dual function applications," in IEEE Transactions on Microwave Theory and Techniques, vol. 56, no. 12, pp. 3079-3085, 2008.

[18] A. K. Agrawal and E. L. Holzman, "Active phased array design for high reliability," IEEE Transactions on Aerospace and Electronic Systems, vol. 35, no. 4, pp. 1204-1211, 1999.

[19] N. Khandelwal and R. W. Jackson, "Active antenna module for low-cost electronically scanned phased arrays," IEEE Transactions on Microwave Theory and Techniques, vol. 56, no. 10, pp. 2286-2292, 2008.

[20] J. J. Schuss, J. Upton, B. Myers, et al., "The IRIDIUM main mission antenna concept," IEEE Transactions on Antennas and Propagation, vol. 47, no. 3, pp. 416-424, 1999. 

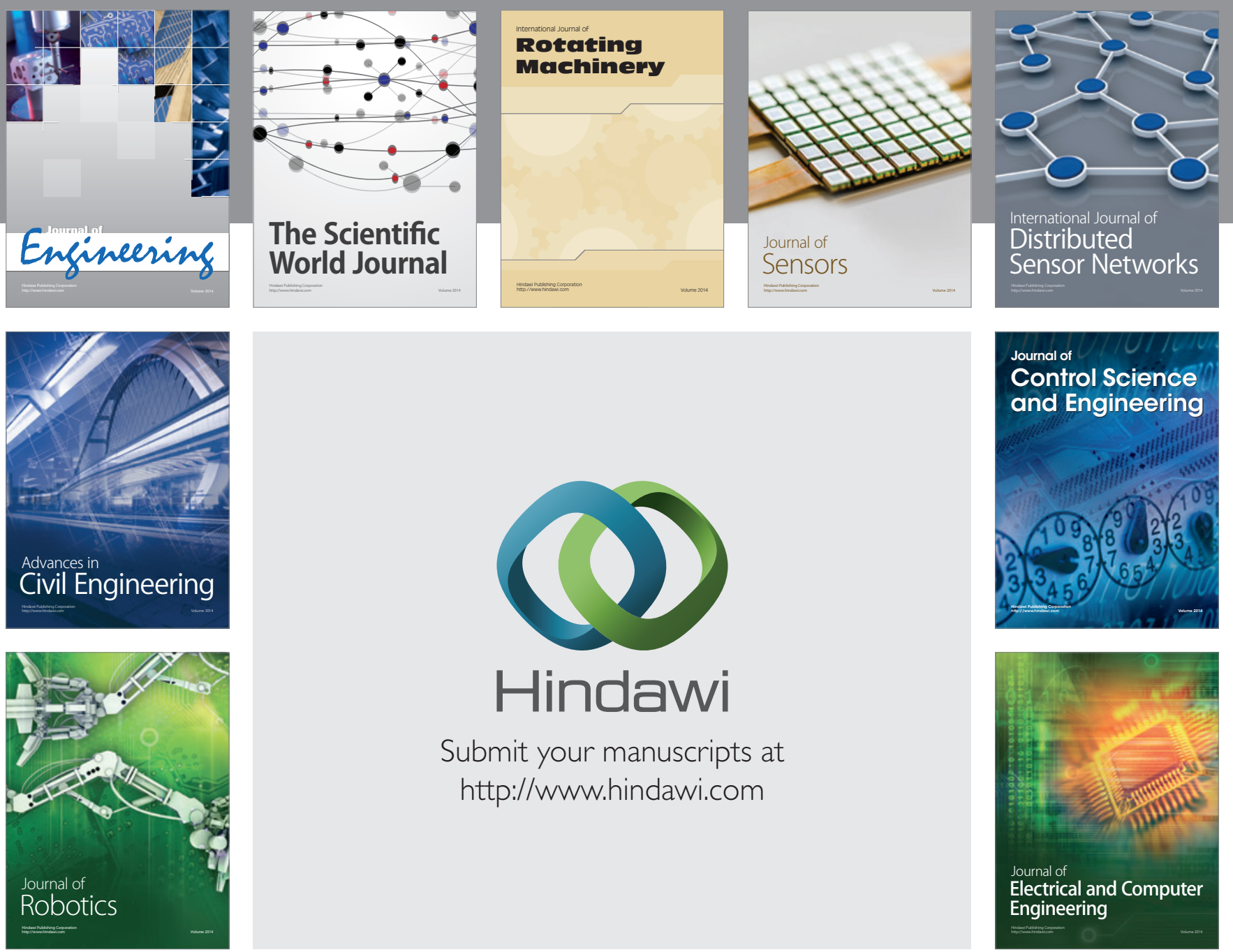

Submit your manuscripts at

http://www.hindawi.com
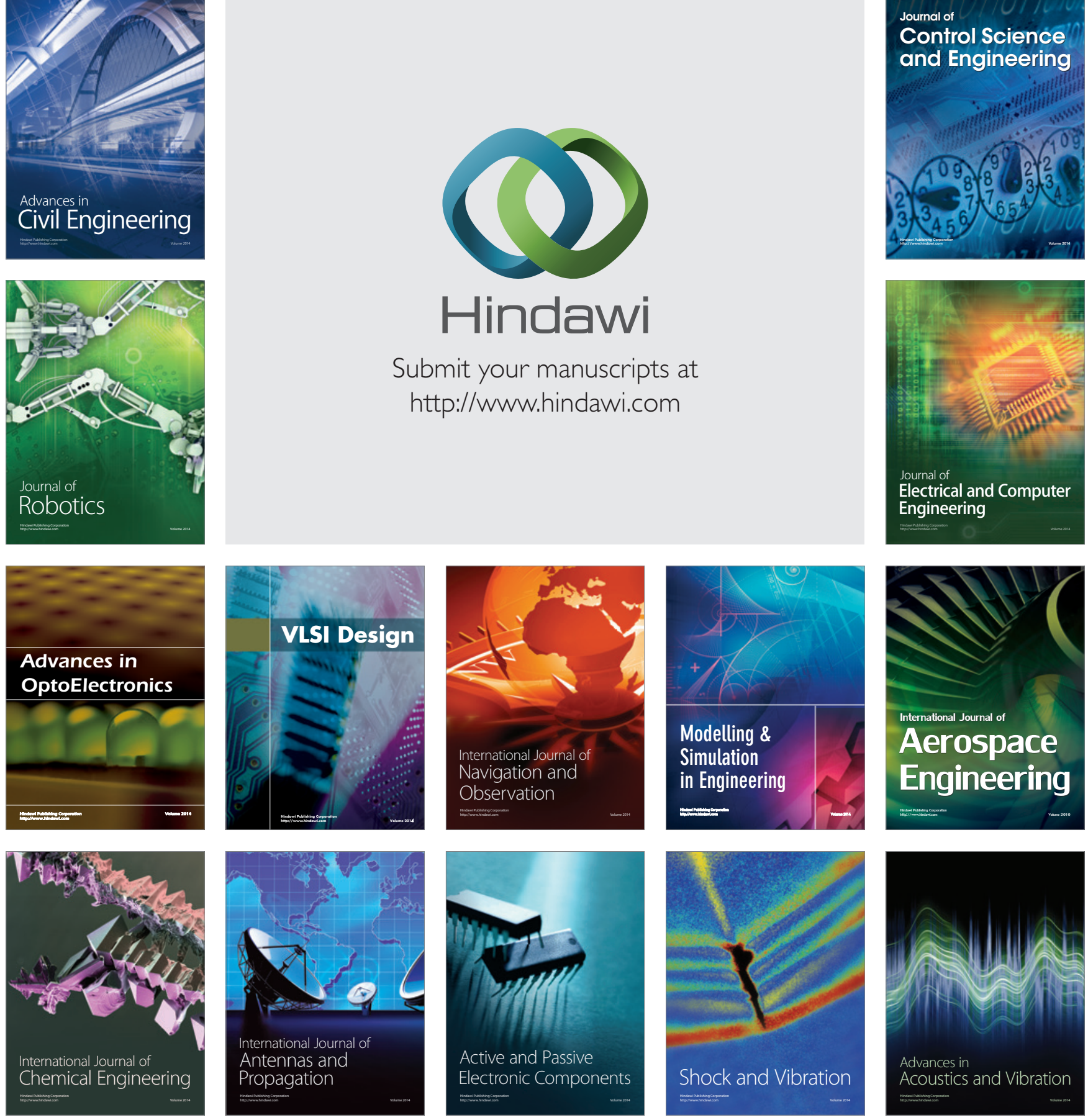